\title{
Low-voltage STEM-EELS Quantification for Beam Sensitive Material Characterization
}

\author{
Nicolas Dumaresq, Nicolas Brodusch and Raynald Gauvin \\ McGill University, Montreal, Quebec, Canada
}

Scanning transmission electron microscopy (STEM) has been proven to be a powerful tool for the study of microstructure and morphology with subnanometer resolution. Furthermore, a STEM paired with electron energy loss spectroscopy can provide useful information on the specimen elementary composition. However, these techniques are normally used with high electron beam voltage (80-200 keV) which induce knock-on beam damage into low $\mathrm{Z}$ materials. This becomes problematic when the microstructure of a low $\mathrm{z}$ material such as a lithium ion battery (LIB) is observed since the change into the material cause by the beam damage is close to the changes cause by the use of batteries during cycling.

To prevent beam damage such as knock-on damage, it is well known that the beam energy needs to be below the energy threshold of atomic displacement. [1,2] Computational calculation such as density functional theory (DFT) showed that for low Z elements such as boron and nitrogen in $\mathrm{h}-\mathrm{BN}$, the threshold energies for atomic displacements are $\sim 19 \mathrm{eV}$ and $\sim 23 \mathrm{eV}[3]$ which correspond to a beam voltage around $80 \mathrm{keV}$. This means that to reduce beam damages on low $\mathrm{Z}$ elements, we need to turn to low beam voltage electron microscopy.

Not only low beam voltage help to reduce beam damage, but it is also a useful tool to image low $\mathrm{Z}$ samples such as carbon base material [4] since the contrast increase due to the more dominant plural scattering. However, elemental identification and quantification in a microscope using low beam voltage still need to be worked on. Electron energy loss spectroscopy (EELS) is a known technique that can give elemental information with higher counts than similar technique such as X-ray spectroscopy with the same electron dose. Which makes EELS a powerful tool that can generate enough counts to do elemental quantification while still working at a low beam voltage. However, a small amount of work has been done on low beam voltage EELS.

The aim of this work is to achieve EELS quantification with a low beam voltage STEM on beam sensitive material. EELS spectra were acquired using a state-of-the-art dedicated transmission scanning electron microscope (Hitachi-SU9000EA) at $30 \mathrm{kev}$. Interesting results will be presented on low-voltage EELS quantification on standard such as $\mathrm{TiC}$ and h-BN using the integration ratio method. 


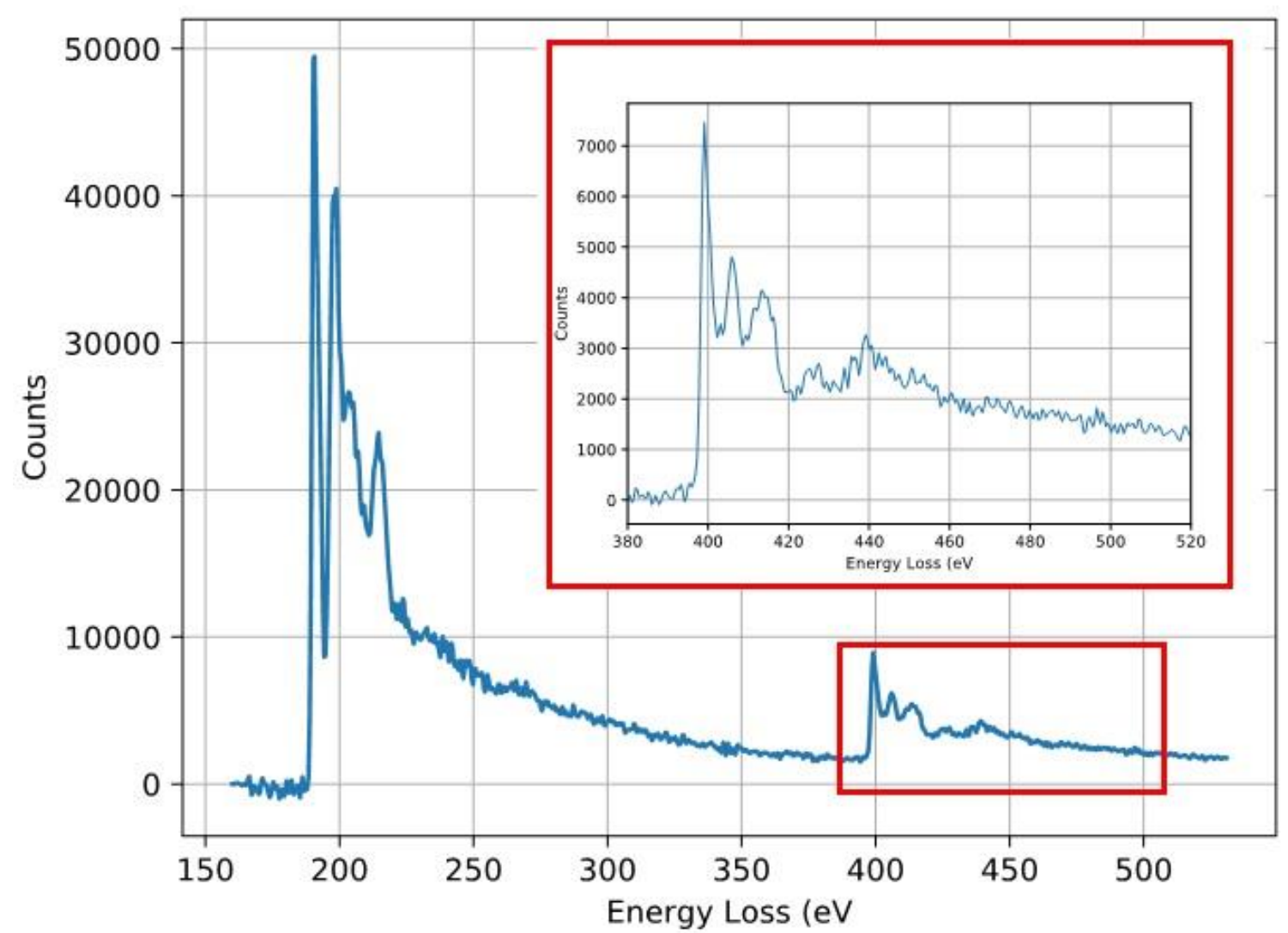

Figure 1. Background-substracted and deconvoluted EELS spectrum showing the boron and nitrogen Kedges of a h-BN standard acquired with the Hitachi-SU9000EA at $30 \mathrm{kV}$ 


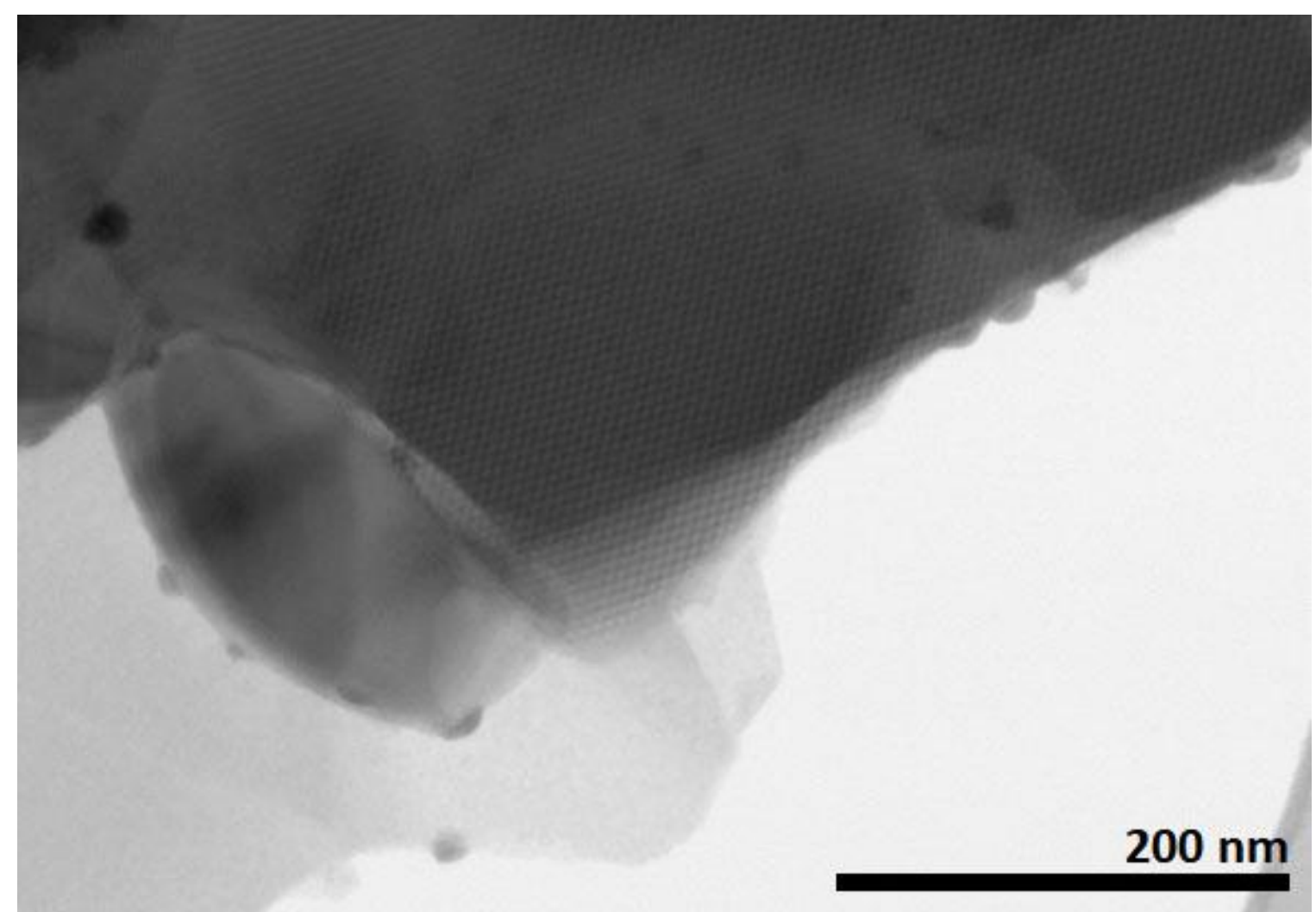

Figure 2. BF-STEM images of a h-BN standard acquired with the Hitachi-SU9000EA at $30 \mathrm{keV}$ where moire fringes are exhibited

\section{References}

1. Williams, D.B. and C.B. Carter, Inelastic Scattering and Beam Damage, in Transmission Electron Microscopy: A Textbook for Materials Science. 2009, Springer US: Boston, MA. p. 53-71.

2. Egerton, R.F., Radiation damage to organic and inorganic specimens in the TEM. Micron, 2019. 119: p. $72-87$.

3. Kotakoski, J., et al., Electron knock-on damage in hexagonal boron nitride monolayers. Physical Review B, 2010. 82(11): p. 113404.

4. Suenaga, K., et al., Visualizing and identifying single atoms using electron energy-loss spectroscopy with low accelerating voltage. Nature Chemistry, 2009. 1(5): p. 415-418. 